\title{
BMJ
}

\section{Mother to child transmission of HIV among Zimbabwean women who seroconverted postnatally: prospective cohort study}

\begin{abstract}
Jean H Humphrey, associate professor of international health, ${ }^{1,2}$ Edmore Marinda, senior statistician, ${ }^{1,3}$ Kuda Mutasa, laboratory manager, ${ }^{1}$ Lawrence $\mathrm{H}$ Moulton, professor of international health, ${ }^{2}$ Peter $\mathrm{I}$ lliff, medical director, ${ }^{1,4}$ Robert Ntozini, senior statistician, ${ }^{1}$ Henry Chidawanyika, data manager, ${ }^{1}$ Kusum J Nathoo, professor of paediatrics and child health, ${ }^{3}$ Naume Tavengwa, senior counselling adviser, ${ }^{1}$ Alison Jenkins, deputy director, ${ }^{1}$ HIV behaviour change communication technical adviser, ${ }^{5}$ Ellen G Piwoz, director, Center for Nutrition, ${ }^{6}$ senior programme officer, ${ }^{7}$ Philippe Van de Perre, professor of bacteriology and virology, ${ }^{8}$ Brian J Ward, professor of infectious diseases and microbiology ${ }^{9}$ on behalf of the ZVITAMBO study group
\end{abstract}

\section{ABSTRACT}

ZVITAMBO Project, Harare,

Zimbabwe

${ }^{2}$ Department of International Health, Johns Hopkins Bloomberg School of Public Health, Baltimore, MD, USA

${ }^{3}$ School of Public Health, Faculty of Health Sciences, University of the Witwatersrand, Johannesburg, South Africa

${ }^{4}$ Department of Paediatrics and Child Health, University of Zimbabwe, Harare, Zimbabwe

${ }^{5}$ Population Services International, Kigali, Rwanda

${ }^{6}$ Academy for Educational Development, Washington, DC, USA

${ }^{7}$ Bill and Melinda Gates Foundation, Seattle, WA, USA

${ }^{8}$ Laboratory of BacteriologyVirology, Montpellier University Hospital, Montpellier, France

${ }^{9}$ The Research Institute of the McGill University Health Centres, Montreal, Quebec, Canada Correspondence to: J H Humphrey, ZVITAMBO Project, No 1 Borrowdale Road, Borrowdale, Harare, Zimbabwe jhumphrey@zvitambo.co.zw

Cite this as: BMJ 2010;341:c6580 doi:10.1136/bmj.c6580
Objectives To estimate the rates and timing of mother to infant transmission of HIV associated with breast feeding in mothers who seroconvert postnatally, and their breast milk and plasma HIV loads during and following seroconversion, compared with women who tested HIV positive at delivery.

Design Prospective cohort study.

Setting Urban Zimbabwe.

Participants 14110 women and infants enrolled in the Zimbabwe Vitamin A for Mothers and Babies (ZVITAMBO) trial (1997-2001).

Main outcome measures Mother to child transmission of HIV, and breast milk and maternal plasma HIV load during the postpartum period.

Results Among mothers who tested HIV positive at baseline and whose infant tested HIV negative with polymerase chain reaction $(P C R)$ at six weeks $(n=2870)$, breastfeeding associated transmission was responsible for an average of 8.96 infant infections per 100 child years of breast feeding ( $95 \% \mathrm{Cl} 7.92$ to 10.14$)$ and varied little over the breastfeeding period. Breastfeeding associated transmission for mothers who seroconverted postnatally $(n=334)$ averaged 34.56 infant infections per 100 child years $(95 \% \mathrm{Cl} 26.60$ to 44.91$)$ during the first nine months after maternal infection, declined to $9.50(95 \% \mathrm{Cl} 3.07$ to 29.47) during the next three months, and was zero thereafter. Among women who seroconverted postnatally and in whom the precise timing of infection was known ( $\leq$ 90 days between last negative and first positive test; $n=51), 62 \%(8 / 13)$ of transmissions occurred in the first three months after maternal infection and breastfeeding associated transmission was 4.6 times higher than in mothers who tested HIV positive at baseline and whose infant tested HIV negative with PCR at six weeks. Median plasma HIV concentration in all mothers who seroconverted postnatally declined from $5.0 \log _{10}$ copies/mL at the last negative enzyme linked immunosorbent assay (ELISA) to $4.1 \log _{10}$ copies $/ \mathrm{mL}$ at
9-12 months after infection. Breast milk HIV load in this group was $4.3 \log _{10}$ copies/mL 0-30 days after infection, but rapidly declined to $2.0 \log _{10}$ copies $/ \mathrm{mL}$ and $<1.5 \log _{10}$ copies/mL by 31-90 days and more than 90 days, respectively. Among women whose plasma sample collected soon after delivery tested negative for HIV with ELISA but positive with PCR ( $n=17), 75 \%$ of their infants were infected or had died by 12 months. An estimated $18.6 \%$ to $20.4 \%$ of all breastfeeding associated transmission observed in the ZVITAMBO trial occurred among mothers who seroconverted postnatally.

Conclusions Breastfeeding associated transmission is high during primary maternal HIV infection and is mirrored by a high but transient peak in breast milk HIV load.

Around two thirds of breastfeeding associated transmission by women who seroconvert postnatally may occur while the mother is still in the "window period" of an antibody based test, when she would test HIV negative using one of these tests.

Trial registration Clinical trials.gov NCT00198718.

\section{INTRODUCTION}

Primary HIV infection (that is, the period between infection and seroconversion, when detectable concentrations of HIV specific antibodies have developed) is characterised by a high viral burden ${ }^{1}$ and high rates of sexual transmission, ${ }^{2}$ which account for a large proportion of all new sexually acquired HIV infections. ${ }^{3}$ Breastfeeding associated mother to child transmission of HIV is also known to be high among women who acquire HIV infection postnatally, although empirical data on the rates of breastfeeding associated transmission during primary infection are limited. In a summary of five reports that together included 42 women who seroconverted while breast feeding, ${ }^{4-8} 12(29 \%)$ women transmitted HIV to their infants. ${ }^{9}$ Five of these cases were part of a cohort in Rwanda studied by Van de Perre and colleagues. This group documented that four of the five 
transmissions had occurred within three months of maternal seroconversion and concluded that breastfeeding associated transmission of HIV is greatly facilitated by the peak viraemia associated with primary $\mathrm{HIV}$ infection. More recently in a retrospective case series from China, $38(35.8 \%)$ of 106 women who acquired HIV infection through a postnatal blood transfusion infected their infants via breast feeding. ${ }^{10}$

The Zimbabwe Vitamin A for Mothers and Babies (ZVITAMBO) trial enrolled 14110 postpartum mothers and their infants within 96 hours after delivery (baseline) between 1997 and 2000. ${ }^{112}$ The primary objectives of the trial were to determine the effect of maternal, neonatal, or maternal and neonatal vitamin A supplementation on the incidence of HIV infection among postpartum women, the incidence of breastfeeding associated transmission of HIV, and infant mortality. Among mothers who were HIV positive at baseline, vitamin A had no effect on breastfeeding associated transmission. ${ }^{11}$ However, early exclusive breast feeding (feeding only breast milk) was associated with a $75 \%$ reduction in breastfeeding associated transmission at six months compared with early mixed breast feeding (feeding both breast milk and non-breast milk liquid or solid foods). ${ }^{12}$ Among women who were HIV negative at baseline, 351 seroconverted postnatally. ${ }^{13}$

Motivated in part by a World Health Organization technical consultation on infant feeding and HIV, ${ }^{14}$ which identified the "risk of postnatal mother to child transmission in women who seroconvert during lactation" as a key area for future research, we conducted the current analysis of ZVITAMBO data and archived specimens to estimate the rates and timing of mother to child transmission for these women who seroconverted postnatally, to determine their breast milk and plasma HIV loads during and after seroconversion, and to compare these findings with those of women enrolled in the ZVITAMBO trial who tested HIV positive at baseline.

\section{METHODS}

Between November 1997 and January 2000, mothers and their newborn infants at 14 maternity clinics and hospitals in greater Harare, Zimbabwe, were enrolled within 96 hours of delivery. ${ }^{11}{ }^{12}$ Mother-infant pairs were eligible if the infant was a singleton with birth weight greater than or equal to $1500 \mathrm{~g}$, the mother planned to stay in Harare after delivery, and neither mother nor infant had an acutely life threatening condition. Mothers provided written informed consent. Detailed description of eligibility and consent rates has been previously published. ${ }^{15}$

Baseline characteristics were collected by questionnaire or from hospital records. Maternal blood and breast milk, and infant blood, were collected at baseline. Aliquots of maternal plasma and breast milk supernatant, and infant plasma and cell pellets (Amplicor whole blood polymerase chain reaction (PCR) sample preparation method; Roche Diagnostic Systems, Alameda, CA), were archived at $-70{ }^{\circ} \mathrm{C}$. Baseline maternal HIV status was determined by two enzyme linked immunosorbent assays (ELISAs) run in parallel; discordant ELISA results were repeated and then resolved by western blot if necessary.

Follow-up was conducted at six weeks, three months, and then three monthly for 12 to 24 months. For all HIV positive mothers and their infants, and a representative subsample of HIV negative women and their infants (52\% of total), maternal blood and breast milk, and infant blood, were collected and archived at all visits. For the remaining $48 \%$ of women who were HIV negative at baseline and their infants, maternal samples were archived at six and 12 months, and infant blood at 12 months. Women who were HIV negative at baseline were retested for HIV at all subsequent blood collections. Cell pellets were archived at all time points for infants of HIV positive mothers and at the time of the first positive maternal ELISA and at all subsequent infant blood collections for infants whose mothers seroconverted postnatally.

Among women who seroconverted during followup but had a last negative ELISA test less than three months post partum, late prenatal infections could not be distinguished from early postnatal infections on the basis of ELISA results because the interval between infection and seroconversion can be up to three months. This group included women who tested negative with ELISA at baseline and then either tested positive with ELISA at six weeks or did not provide blood samples before testing positive at three months or later. To further characterise timing of infection for these women, archived baseline plasma samples were assayed for HIV load by quantitative HIV RNA testing (Roche Amplicor HIV-1 Monitor test, version 1.5 Roche Diagnostic Systems).

HIV testing for exposed infants was conducted after all patient contact was completed. In the infants of mothers who tested HIV positive at baseline, the last available infant specimen was tested by ELISA for plasma samples collected at 18 months or later (GeneScreen HIV 1/2; Sanofi Diagnostics Pasteur, Johannesburg, South Africa) or by PCR assay for cell pellets collected at less than 18 months (Roche Amplicor HIV-1 Monitor test, version 1.5). If negative, the infant was classified as uninfected; if positive, samples collected at earlier time points were tested to establish the timing of infection. All available specimens from infants of women who seroconverted were tested for HIV by PCR.

For mothers who seroconverted, breast milk supernatant and plasma samples collected at the time of the mother's last negative ELISA test and at all subsequent time points were tested for HIV load using the ultrasensitive (milk) and standard (plasma) methods (detection limits 50 and 400 copies/mL, respectively). Plasma and breast milk HIV load was also measured for a representative sample of women who tested HIV positive at baseline.

Infant feeding histories were ascertained and breastfeeding exclusivity defined as previously described. ${ }^{12}$ Throughout the trial, medical care, counselling, ${ }^{16}{ }^{17}$ and condoms were freely provided. Education and 
counselling of HIV negative women included HIV prevention strategies and the message that maternal seroconversion during breast feeding is associated with an increased risk of breastfeeding associated transmission. Antenatal HIV testing, antiretroviral prophylaxis for prevention of mother to child transmission, and highly active antiretroviral therapy were not available in public sector facilities during the time of the trial.

\section{Statistical analysis}

Women who ever tested HIV positive and their infants were classified into four groups according to the risk period for mother to child transmission:

1 Baseline positive: mother tested HIV positive at baseline with ELISA or western blot (mother infected before delivery; infant at risk of intrauterine, intrapartum, and breastfeeding associated transmission);

2 Baseline positive and at risk of breastfeeding associated transmission (baseline positive at risk of BFT): mother tested HIV positive with ELISA or western blot at baseline and her infant tested negative with PCR at six weeks (mother infected before delivery; infant escaped intrauterine and intrapartum infection but at risk of breastfeeding associated transmission);

3 Postnatal seroconverter: mother tested HIV negative with ELISA or western blot at three months postpartum or later or her baseline plasma had undetectable HIV RNA, and mother tested positive with ELISA or western blot at a subsequent visit (mother seroconverted postnatally; infant at risk of breastfeeding associated transmission after maternal infection while still breast feeding);

4 Baseline seroconverter: mother's baseline plasma sample tested HIV negative with ELISA or western blot but the same sample positive with RNA PCR (mother infected during late pregnancy and was seroconverting during delivery; infant at risk of late intrauterine, intrapartum, and breastfeeding associated transmission).

We compared mother to child transmission rates for seroconverting women with women who had tested HIV positive at baseline and whose infants were at risk of HIV infection during the same periods. Breastfeeding associated transmission rates were compared between the baseline positive at risk of BFT group and the postnatal seroconverter group because the infants in both groups were at risk of breastfeeding associated transmission only. Total transmission rates were compared between the baseline seroconverter group and the baseline positive group because these women's infants were at risk of intrauterine, intrapartum, and breastfeeding associated transmission. Baseline characteristics were compared between the baseline positive at risk of BFT group and the postnatal seroconverter group, and between the baseline positive group and the baseline seroconverter group. Differences were tested by Kruskal-Wallis tests or $\chi^{2}$ tests, as appropriate.
Kaplan-Meier methods were used to estimate breastfeeding associated transmission rates for baseline positive at risk of BFT pairs and postnatal seroconverter pairs, with timelines beginning at six weeks post partum for baseline positive at risk of BFT mothers and at maternal HIV infection (midpoint between last negative and first positive tests) for postnatal seroconverters. Among women in the postnatal seroconverter group, the median number of days (interquartile range) between the mother's last negative and first positive ELISA tests (hereafter termed "seroconversion interval") was 181 (range 92 to 323) days. Thus, for some mothers, our estimated time of infection was relatively imprecise. To more closely examine the temporal relation between maternal infection and breastfeeding associated transmission, we repeated the analysis restricting postnatal seroconverter mothers to those with seroconversion intervals of less than or equal to 90 days (hereafter termed "short seroconversion interval"). Kaplan-Meier methods were also used to estimate and compare rates of total transmission and total transmission or death for infants of baseline positive and baseline seroconverter mothers.

In all Kaplan-Meier analyses, infants who never had a positive HIV test were censored at the time of their last negative result, 60 days after breastfeeding cessation or their mother's date of death, or at 24 months of age, whichever occurred first. Differences were tested using the log rank test. Smoothed hazard functions were calculated using an Epanechnikov kernel function in Stata.

We calculated rates of breastfeeding associated transmission per 100 child years of breast feeding for three month intervals over the first 12 months of HIV exposure. These intervals represented time after six weeks of age for infants of baseline positive at risk of BFT women and time after maternal HIV infection for infants of postnatal seroconverter women. We examined whether infant age at the time of maternal infection modified the effect of maternal seroconversion on breastfeeding associated transmission, first by Poisson regression and then with a Cox model that included child age at maternal primary infection stratified in three month intervals; transmissions per 100 child years of breastfeeding exposure were calculated for each stratum.

Concentrations of breast milk and plasma HIV load were $\log _{10}$ transformed. Among baseline positive at risk of BFT women, we calculated the proportions of plasma and breast milk samples with detectable virus loads, and the median (intra(?er?)quartile range) HIV load among detectable samples for each specimen type at each visit during the postpartum year. For postnatal seroconverter women, we calculated the same statistics for available plasma and breast milk samples collected at the time of the mother's last negative ELISA test and at time points less than 30 days, 31-90 days, 91-180 days, 181-270 days, and 271-365 days after maternal infection. 


\section{RESULTS}

Study population

Of the 14110 mothers enrolled in the ZVITAMBO trial, $4495(32 \%)$ tested HIV positive at baseline according to ELISA or western blot (classified as baseline positive; fig 1). A total of 351 women seroconverted during follow-up; 205 had their last negative ELISA or western blot test at or later than three months post partum (classified as postnatal seroconverter). Of the remaining 146 women who had their last negative ELISA or western blot test at less than three months post partum, RNA HIV was undetectable in baseline plasma samples from 129 women (classified as postnatal seroconverters) and detectable for 17 women (classified as baseline seroconverters). Among infants born to mothers who were HIV positive at baseline $(\mathrm{n}=4495), 2870$ were alive and tested negative for HIV with PCR at six weeks (classified as baseline positive at risk of BFT).

Women classified as postnatal seroconverters were younger, less likely to be married or die during the postpartum year, and had lower parity, higher CD4 cell count, higher haemoglobin concentration, and more years of schooling than the baseline positive at risk of BFT women (table 1). Women in the baseline positive and baseline seroconverter groups did not differ significantly on any baseline characteristic examined. Breastfeeding duration was prolonged among all women, but somewhat longer among women who were HIV negative at baseline compared with women who were HIV positive at baseline (median (IQR) weaning age 578 (515-661) and 548 (486-639) days, respectively). However, among women still breast feeding, the frequency of breast feeding per 24 hours (that is, breastfeeding intensity) did not differ between HIV positive women and HIV negative women at any follow-up visit (data not presented). Exclusive breast feeding - even to three months - was rare among all women (table 1).

\section{Breastfeeding associated transmission among baseline} positive at risk of BFT pairs and postnatal seroconverter pairs During the first 12 months of exposure, breastfeeding associated transmission occurred in $8.5 \%$ of baseline positive at risk of BFT mother-baby pairs, $23.6 \%$ of all postnatal seroconverter pairs, and $30.2 \%$ of the 51 postnatal seroconverter pairs with a short ( $\leq 90$ days) maternal seroconversion interval (fig 2; table 2). During the second year of exposure, there was no further transmission by postnatal seroconverter mothers but transmission continued among the baseline positive at risk of BFT women so that by 24 months post partum $14.2 \%$ had transmitted HIV to their infants. Breastfeeding associated transmission was significantly higher among baseline positive at risk of BFT women than in both the group of all postnatal seroconverter women $\left(\chi^{2}=41.7 ; \mathrm{P}<0.001\right)$ and the subgroup of postnatal seroconverters with a short seroconversion interval $\left(\chi^{2}=30.9 ; \quad \mathrm{P}<0.001\right)$. Within the postnatal seroconverters group, breastfeeding associated transmission was higher among those with a short seroconversion interval than among all postnatal seroconverters. However, this difference was not significant, probably owing to the small number of women in the short seroconversion subgroup $\left(\chi^{2}=2\right.$; $\mathrm{P}=0.16)$.

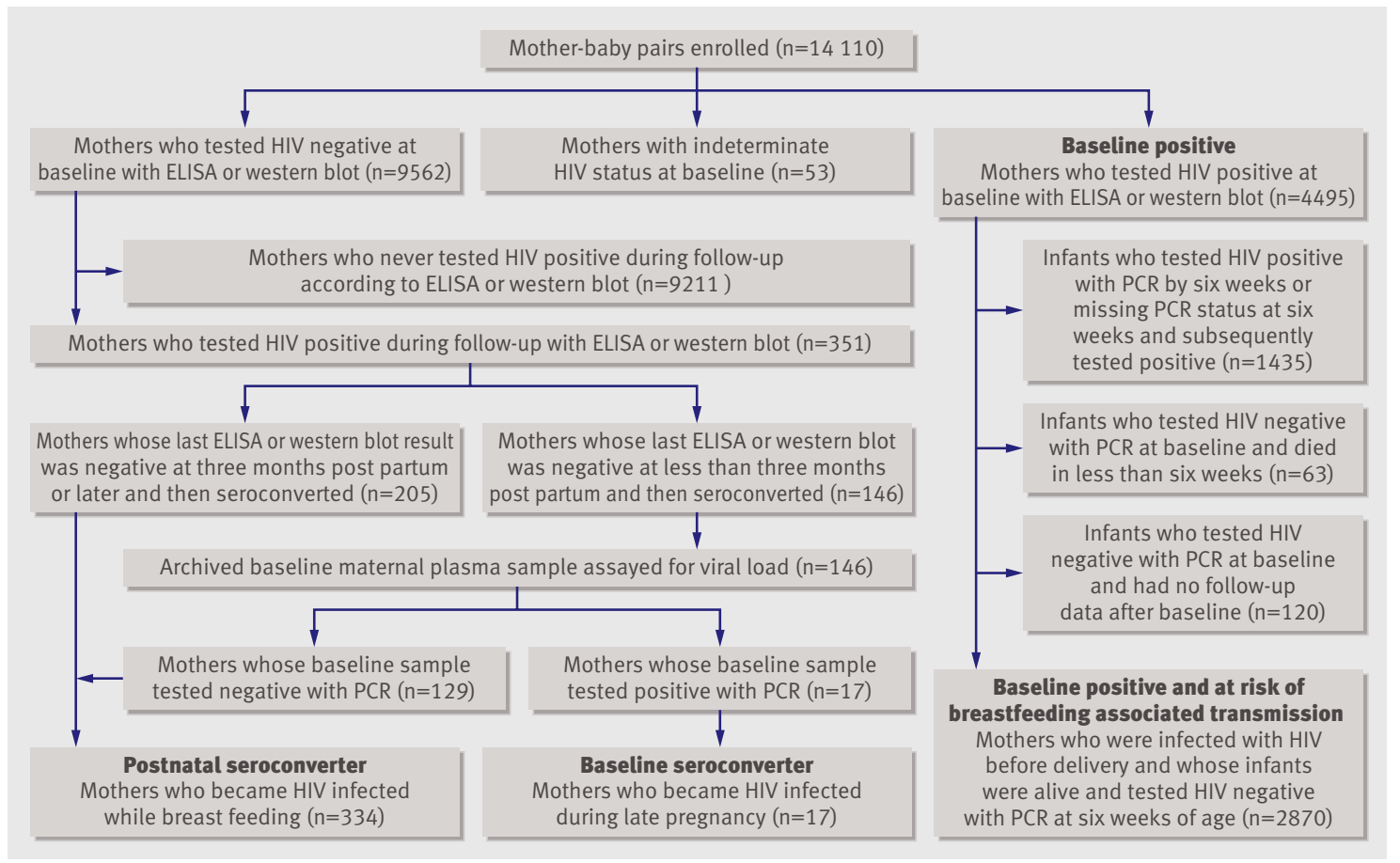

Fig 1 Classification of HIV infected mothers according to mother to child risk of transmission. ELISA, enzyme linked immunosorbent assay; $\mathrm{PCR}$, polymerase chain reaction 
Table 1|Baseline characteristics of all mothers and their infants according to timing of maternal HIV infection*

\begin{tabular}{|c|c|c|c|c|c|c|}
\hline & $\begin{array}{l}\text { Baseline positive } \\
\qquad(\mathrm{n}=4495) \dagger\end{array}$ & $\begin{array}{c}\text { Baseline } \\
\text { seroconverter } \\
(n=17) \dagger\end{array}$ & Pvalue $\ddagger$ & $\begin{array}{l}\text { Baseline positive and at } \\
\text { risk of breastfeeding } \\
\text { associated } \\
\text { transmission } \\
(n=2870) \dagger\end{array}$ & $\begin{array}{l}\text { Postnatal } \\
\text { seroconverter } \\
(n=336) \dagger\end{array}$ & P value $\ddagger$ \\
\hline \multicolumn{7}{|l|}{ Infant characteristics } \\
\hline Birth weight (kg; mean (SD)) & $2.92(0.5)$ & $2.85(0.4)$ & 0.55 & $2.93(0.46)$ & $2.96(0.48)$ & 0.74 \\
\hline Sex (proportion boys (\%)) & 50.8 & 52.9 & 0.86 & 51.1 & 48.8 & 0.42 \\
\hline Gestational age (weeks; mean (SD)) & $39.1(1.5)$ & $39.0(1.6)$ & 0.70 & $39.1(1.5)$ & $39.2(1.5)$ & 0.87 \\
\hline Caesarean delivery (\%) & 8.7 & 5.9 & 1.00 & 9.4 & 9.3 & 0.96 \\
\hline $\begin{array}{l}\text { Time from membrane rupture to } \\
\text { delivery more than } 4 \text { hrs (\%) }\end{array}$ & 39.2 & 43.8 & 0.80 & 38.0 & 41.4 & 0.24 \\
\hline $\begin{array}{l}\text { Exclusive breastfeeding to } \geq 3 \text { months } \\
(\mathrm{n} / \mathrm{N}(\%))\end{array}$ & $202 / 2763(7.31)$ & $0 / 11(0)$ & 0.28 & $156 / 2071(7.69)$ & $13 / 170(7.65)$ & 0.90 \\
\hline \multicolumn{7}{|l|}{ Maternal characteristics } \\
\hline Age (years; mean (SD)) & $25.5(5.2)$ & $25.2(5.2)$ & 0.82 & $25.6(5.1)$ & $23.3(4.7)$ & $<0.0001$ \\
\hline Parity (mean (SD)) & $2.3(1.2)$ & $2.0(1.2)$ & 0.37 & $2.3(1.2)$ & $1.9(1.1)$ & $<0.0001$ \\
\hline \multicolumn{7}{|l|}{ Marital status (n (\%)) } \\
\hline Married or in a stable union & 3771 (91.9) & $16(94.1)$ & 0.76 & $2650(92.1)$ & $295(88.6)$ & $0.003 \S$ \\
\hline Single or never married & $183(4.5)$ & $1(5.9)$ & - & $131(4.6)$ & $31(9.3)$ & - \\
\hline Widowed or divorced & $149(3.7)$ & 0 & - & $96(3.3)$ & $7(2.1)$ & - \\
\hline \multicolumn{7}{|l|}{ CD4 count (cells $\left.\times 10^{6} / \mathrm{L}\right)$} \\
\hline$n$ & 3907 & - & - & 2521 & 28 & - \\
\hline Median (IQR) & $400(258-569)$ & - & - & $418(276-88)$ & $898(561-1043)$ & $<0.0001$ \\
\hline \multicolumn{7}{|l|}{ Viral load ( $\log _{10}$ copies/mL) } \\
\hline $\mathrm{n}$ & 406 & - & - & 277 & - & - \\
\hline Median (IQR) & $4.01(3.46-4.59)$ & - & - & $3.90(3.36-4.42)$ & - & - \\
\hline \multicolumn{7}{|l|}{ Haemoglobin concentration $(\mathrm{g} / \mathrm{L})$} \\
\hline $\mathrm{n}$ & 2527 & 6 & - & 1588 & 169 & - \\
\hline Mean (SD) & $111.1(19.5)$ & $120.3(21.4)$ & 0.25 & $111.2(19.0)$ & $117.1(21.6)$ & 0.006 \\
\hline $\begin{array}{l}\text { Mid-upper arm circumference }(\mathrm{cm} \text {; } \\
\text { mean (SD)) }\end{array}$ & $25.7(2.9)$ & $25.9(2.2)$ & 0.70 & $25.8(2.9)$ & $25.9(2.9)$ & 0.79 \\
\hline Education (years; mean (SD)) & $9.5(2.0)$ & $9.9(1.7)$ & 0.36 & $9.5(2.0)$ & $9.7(1.7)$ & 0.05 \\
\hline $\begin{array}{l}\text { Husband's education (years; mean } \\
\text { (SD)) }\end{array}$ & $12.7(11.8)$ & $14.7(16.0)$ & 0.60 & $12.9(12.3)$ & $13.8(13.8)$ & 0.24 \\
\hline \multicolumn{7}{|l|}{ Income (\$US/month) } \\
\hline $\mathrm{n}$ & 3036 & 12 & - & 2120 & 236 & - \\
\hline Median (IQR) & $76.4(46.8-135.3)$ & $77.6(57.8-131.6)$ & 0.72 & $76.7(47.5-138.7)$ & $78.8(52.4-151.5)$ & 0.08 \\
\hline Death within 12 months (n (\%)) & $146(4.4)$ & 0 & $1.00 \S$ & $62(2.7)$ & $1(0.4)$ & $0.04 \S$ \\
\hline
\end{tabular}

Among baseline positive at risk of BFT pairs, the instantaneous (one day) probability of breastfeeding associated transmission was relatively constant at $\sim 2.5 \times 10^{-4}$ throughout breastfeeding exposure (fig 2, lower panel). For all postnatal seroconverter pairs, this probability peaked at $11.8 \times 10^{-4}$ at 70 days after maternal infection, declining to $\sim 3 \times 10^{-4}$ by nine months after maternal infection. Among postnatal seroconverter women with a short seroconversion interval, the probability of breastfeeding associated transmission peaked higher $\left(18.5 \times 10^{-4}\right)$ and declined more precipitously.

Breastfeeding associated transmission between six weeks and 24 months among baseline positive at risk of BFT pairs was 8.96 infections per 100 child years of breast feeding (95\% CI 7.92 to 10.14), and varied little over this period (table 3). Conversely, among all postnatal seroconverter pairs, breastfeeding associated transmission averaged 34.56 infections per 100 child years (95\% CI 26.60 to 44.91$)$ during the first nine months after maternal infection, declined to 9.50 (95\% CI 3.07 to 29.47) during the next three months, and was zero thereafter. Among postnatal seroconverter women with a short seroconversion interval, $62 \%$ $(8 / 13)$ of transmissions occurred in the first three months after maternal infection (77.62 infections/100 child years, $95 \%$ CI 38.82 to 155.21 ) and all remaining transmissions occurred during the next six months, 
Table 2 | Proportion of children infected at specific time points after HIV exposure began according to timing of maternal infection

\begin{tabular}{|c|c|c|c|c|c|c|}
\hline & 30 days & 90 days & 180 days & 270 days & 360 days & 720 days \\
\hline $\begin{array}{l}\text { Baseline positive and at risk } \\
\text { of breastfeeding associated } \\
\text { transmission }(n=2870)\end{array}$ & $\begin{array}{c}1.50 \text { (1.10 to } \\
2.05)\end{array}$ & $\begin{array}{c}2.26(1.75 \text { to } \\
2.91)\end{array}$ & $\begin{array}{c}4.36(3.63 \text { to } \\
5.24)\end{array}$ & $\begin{array}{c}6.52(5.61 \text { to } \\
7.58)\end{array}$ & $\begin{array}{c}8.52(7.44 \text { to } \\
9.74)\end{array}$ & $14.17(12.18$ to 16.44$)$ \\
\hline $\begin{array}{l}\text { Postnatal seroconverter } \\
(n=334)\end{array}$ & $\begin{array}{c}1.74 \text { (0.73 to } \\
4.13)\end{array}$ & $\begin{array}{c}9.42(6.56 \text { to } \\
13.44)\end{array}$ & $\begin{array}{c}14.31(10.70 \text { to } \\
19.01)\end{array}$ & $\begin{array}{c}21.74 \text { (17.10 to } \\
27.41)\end{array}$ & $\begin{array}{c}23.59(18.67 \text { to } \\
29.55)\end{array}$ & 23.59 (18.67 to 29.55$)$ \\
\hline $\begin{array}{l}\text { Subgroup with } \\
\text { seroconversion interval } \leq \\
90 \text { davs }(n=51)\end{array}$ & $\begin{array}{c}10.64(4.57 \text { to } \\
23.69)\end{array}$ & $\begin{array}{c}17.08(8.93 \text { to } \\
31.26)\end{array}$ & $\begin{array}{c}24.18(14.15 \text { to } \\
39.48)\end{array}$ & $\begin{array}{c}30.17 \text { (18.56 to } \\
46.62)\end{array}$ & $\begin{array}{c}30.17(18.56 \text { to } \\
46.62)\end{array}$ & 30.17 (18.56 to 46.62$)$ \\
\hline
\end{tabular}

All values are percentages $(95 \% \mathrm{Cl})$. Baseline positive and at risk of breastfeeding associated transmission: mother tested HIV positive with ELISA or western blot at baseline and her infant tested negative with polymerase chain reaction (PCR) at six weeks; Postnatal seroconverter: mother tested HIV negative with ELISA or western blot at three months or more post partum or her baseline plasma had undetectable HIV RNA, and mother then tested positive with ELISA or western blot at a subsequent visit.
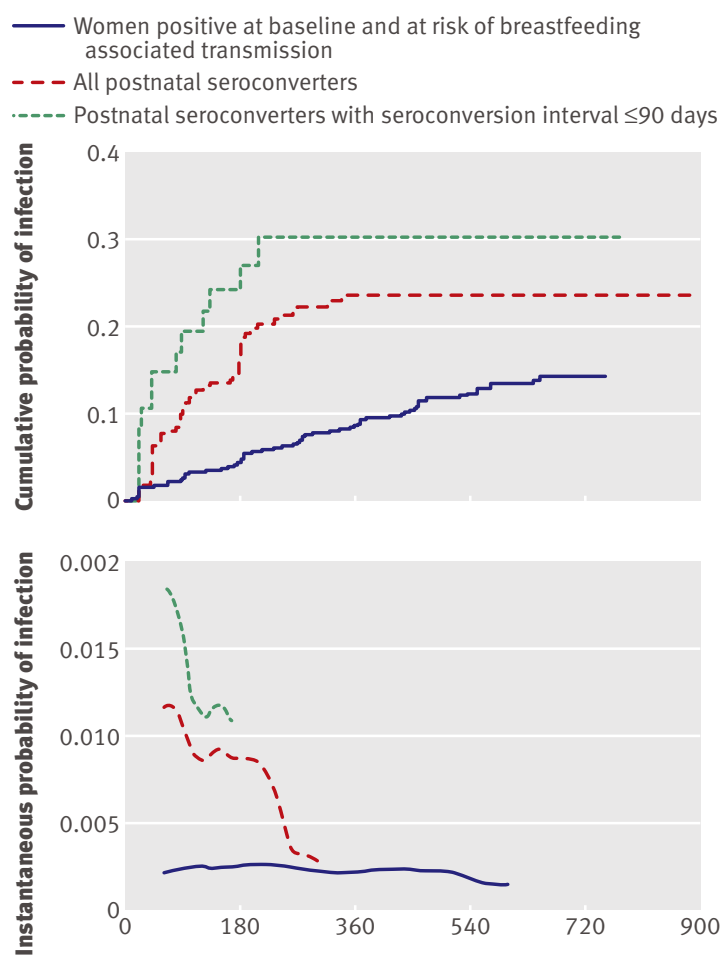

Days of HIV exposure

Fig 2 | Cumulative (top panel) and instantaneous (one day; bottom panel) probability of breastfeeding associated transmission of HIV for three groups of women: those who were positive at baseline and at risk of breastfeeding associated transmission (baseline positive at risk of BFT); all women who seroconverted postnatally; and a subgroup of women who seroconverted postnatally and had a seroconversion interval of less than or equal to 90 days. Time line in the $x$ axis is the time (in days) since HIV exposure began for the infants in each group: six weeks post partum for baseline positive at risk of BFT women; and time of maternal HIV infection (defined as the midpoint of the mother's last negative and first positive ELISA tests) for postnatal seroconverter mothers

averaging 37.92 infections per 100 child years $(95 \%$ CI 15.78 to 91.10$)$. Overall, breastfeeding associated transmission was 2.9 and 4.6 times higher among all postnatal seroconverters and postnatal seroconverters with short seroconversion interval, respectively, than baseline positive at risk of BFT pairs.
Infants of postnatal seroconverter mothers who were younger at the time of their mother's infection were at higher risk of breastfeeding associated transmission than those who were older $(\mathrm{P}<0.001$ from Poisson regression). Risk was similar for infants who were aged $0-3,3-6$, and 6-9 months of age at the time of their mother's infection (45 infections in 43663 child days of breast feeding, equal to 37.6 infections/100 child years of breast feeding), which was 3.8 times higher than the risk among infants aged more than or equal to 9 months at the time of maternal infection $(8$ infections in 29597 child days of breast feeding, equal to 9.9 infections/100 child years of breast feeding).

\section{Plasma and breast milk HIV load}

Among postnatal seroconverter women, $19.3 \%$ of plasma samples collected at the time of the last negative ELISA test and $91 \%$ of the plasma samples collected during the first year after infection had detectable levels of virus (table 4). Among plasma samples with detectable concentrations of virus, median HIV load peaked at $5.0 \log _{10}$ copies $/ \mathrm{mL}$ at the time of the last negative ELISA and declined to $4.1 \log _{10}$ copies/mL at $9-12$ months after infection (fig 3). HIV was detected in $15 \%, 50 \%$, and less than $38 \%$ of the breast milk samples collected at the last negative ELISA test, at 0-31 days after infection, and at 31-365 days after infection, respectively. Among samples of breast milk supernatant with detectable concentrations of virus, HIV load peaked in the same range as plasma $(4.3$ $\log _{10}$ copies/mL during the first 30 days after infection), but declined more precipitously and substantially. The median difference in HIV load between 0-30 days after infection and 31-90 days after infection was $+0.05 \log _{10}$ copies $/ \mathrm{mL}(\mathrm{P}=0.45)$ for plasma and $-0.41 \log _{10}$ copies/ $\mathrm{mL}(\mathrm{P}=0.01)$ for breast milk, whereas the differences between 0-30 days and 181-270 days were $-2.31 \log _{10}$ copies $/ \mathrm{mL} \quad(\mathrm{P}<0.01)$ and $-2.97 \quad \log _{10} \quad$ copies $/ \mathrm{mL}$ $(\mathrm{P}<0.01)$ for plasma and breast milk, respectively. For comparison, among baseline positive women, 95\% of plasma samples tested during the postpartum year had detectable levels of virus and the median HIV load was constant at $\sim 4.2 \log _{10}$ copies $/ \mathrm{mL}$ (fig 4 ; table 5). Similarly, $36 \%$ of breast milk samples from baseline positive women had detectable concentrations of virus and the median HIV load was constant at $\sim 2.0 \log _{10}$ copies $/ \mathrm{mL}$. 
Table $3 \mid$ Breastfeeding associated HIV transmission rates per 100 child years of breastfeeding during the first 12 months of HIV exposure according to timing of maternal HIV infection

\begin{tabular}{|c|c|c|c|c|}
\hline & $\begin{array}{l}\text { Number of infant } \\
\text { infections }\end{array}$ & $\begin{array}{l}\text { Child years of breast } \\
\text { feeding }\end{array}$ & $\begin{array}{c}\text { Rate of transmission per } 100 \text { child } \\
\text { years of breast feeding }\end{array}$ & $\begin{array}{l}\text { Rate ratio for each time } \\
\text { period }\end{array}$ \\
\hline \multicolumn{5}{|c|}{ Baseline positive and at risk of breastfeeding associated transmission* $(n=2870)$} \\
\hline 0 to $<3$ months & 58 & 623.60 & 9.30 (7.19 to 12.03$)$ & Ref \\
\hline 3 to $<6$ months & 51 & 576.12 & $8.85(6.73$ to 11.65$)$ & Ref \\
\hline 6 to $<9$ months & 50 & 532.75 & 9.39 (7.11 to 12.38$)$ & Ref \\
\hline 9 to $<12$ months & 42 & 458.31 & $9.16(6.77$ to 12.40$)$ & Ref \\
\hline 12 to $<24$ months & 49 & 598.70 & $8.18(6.18$ to 10.83$)$ & Ref \\
\hline Total 0 to $<12$ months & 201 & 2190.78 & 9.17 (7.99 to 10.54$)$ & Ref \\
\hline Total 0 to $<24$ months & 250 & 2789.48 & $8.96(7.92$ to 10.14$)$ & Ref \\
\hline \multicolumn{5}{|c|}{ Postnatal seroconverterf (all; $\mathrm{n}=334$ ) } \\
\hline 0 to $<3$ months & 27 & 67.65 & 39.91 (27.37 to 58.20$)$ & 4.29 (2.61 to 6.88$)$ \\
\hline 3 to $<6$ months & 13 & 55.26 & $23.52(13.66$ to 40.51$)$ & $2.66(1.33$ to 4.96$)$ \\
\hline 6 to $<9$ months & 16 & 39.12 & $40.90(25.06$ to 66.76$)$ & $4.36(2.32$ to 7.78$)$ \\
\hline 9 to $<12$ months & 3 & 31.57 & 9.50 (3.07 to 29.47$)$ & $1.04(0.21$ to 3.24$)$ \\
\hline 12 to $<24$ months & 0 & 31.47 & 0 & $0.00(0.00$ to 1.49$)$ \\
\hline Total 0 to $<12$ months & 59 & 193.60 & 30.48 (23.61 to 39.33$)$ & 3.32 (2.44 to 4.46$)$ \\
\hline Total 0 to $<24$ months & 59 & 225.07 & 26.21 (20.31 to 33.83$)$ & 2.92 (2.16 to 3.90$)$ \\
\hline \multicolumn{5}{|c|}{ Postnatal seroconverter $\ddagger$ (subgroup; $n=51$ ) } \\
\hline 0 to $<3$ months & 8 & 10.31 & 77.62 (38.82 to 155.21$)$ & 8.34 (3.44 to 17.55$)$ \\
\hline 3 to $<6$ months & 3 & 7.92 & $37.88(12.22$ to 117.44$)$ & $4.28(0.85$ to 13.23$)$ \\
\hline 6 to $<9$ months & 2 & 5.27 & 37.98 (9.50 to 151.85$)$ & 4.04 (0.48 to 15.39$)$ \\
\hline 9 to $<12$ months & 0 & 3.76 & 0 & $0.00(0.00$ to 11.19$)$ \\
\hline 12 to $<24$ months & 0 & 4.38 & 0 & $0.00(0.00$ to 10.69$)$ \\
\hline Total 0 to $<12$ months & 13 & 27.26 & $47.70(27.69$ to 82.14$)$ & $5.20(2.72$ to 9.09$)$ \\
\hline Total 0 to $<24$ months & 13 & 31.64 & 41.09 (23.86 to 70.77$)$ & $4.58(2.41$ to 7.98$)$ \\
\hline
\end{tabular}

*Baseline positive and at risk of breastfeeding associated transmission: mother tested HIV positive with ELISA or western blot at baseline and her infant tested negative with polymerase chain reaction (PCR) at six weeks.

†Postnatal seroconverter: mother tested HIV negative with ELISA or western blot at three months or more post partum or her baseline plasma had undetectable HIV RNA, and mother then tested positive with ELISA or western blot at a subsequent visit.

$\ddagger$ Postnatal seroconverter (subgroup): subgroup of postnatal seroconverter group for whom the seroconversion interval was less than or equal to 90 days.

Breastfeeding associated transmission among baseline seroconverter pairs and baseline positive pairs

Among the 17 infants of baseline seroconverter mothers, three became infected and one died without being tested within the first 3 months of life; two became infected between 3 and 6 months; and two became infected and one died without being tested between 6 and 9 months (fig 5; table 6). Among baseline seroconverter pairs and baseline positive pairs, respectively, the probability of HIV transmission was $65.9 \%$ and $30.5 \%$ (rate ratio $2.72,95 \%$ CI $1.09 \%$ to $5.61 \%$ ), and the probability of transmission or death was $75.0 \%$ and $34.1 \%$ (rate ratio $3.12,95 \%$ CI $1.42 \%$ to $5.94 \%$ ). During the second year of life, there were no further cases of HIV transmission or death among infants of baseline seroconverter mothers, whereas transmission continued among infants of baseline positive mothers until breast feeding ceased. The probability of infection and the probability of infection or death were significantly higher in baseline seroconverter pairs than in baseline positive pairs $\left(\chi^{2}=4.42, \mathrm{P}<0.04\right.$ and $\chi^{2}=6.67, \mathrm{P}<0.01$, respectively).

Proportion of breastfeeding associated transmission attributable to maternal seroconversion

A total of 258 infant infections were observed among the 2870 baseline positive at risk of BFT mothers, 59 infections among the 334 postnatal seroconverter mothers, and seven infections among the 17 baseline seroconverter mothers. We cannot definitively classify timing of transmission in the seven infants infected by baseline seroconverter mothers. Therefore, between 18.6\% (59 transmissions among postnatal seroconverter mothers $/ 59+258$ total postnatal transmission) and $20.4 \% \quad(59+7 / 59+7+258$, where the calculation is repeated assuming that the baseline seroconverter transmission occurred through breast feeding) of all breastfeeding associated transmission occurred among infants whose mothers seroconverted postnatally.

\section{DISCUSSION}

In this population of mother-baby pairs, in which HIV prevalence was $32 \%$ and incidence was 3.5 infections per 100 women years, up to $20 \%$ of all breastfeeding associated HIV transmission occurred among women who seroconverted postnatally. This proportion will be greater in parts of Africa where incidence is higher (for example, in South Africa ${ }^{18}$ and Swaziland, ${ }^{19}$ where rates of 10 and 16.2 infections per 100 women years, respectively, have been reported) and will likely increase when new recommendations on initiation of maternal highly active antiretroviral therapy and infant antiretroviral prophylaxis during breast feeding $^{20}$ are implemented, which will substantially 
Table 4 |Proportion of specimens with detectable levels of HIV RNA, and median (IQR) HIV load, according to estimated time since HIV infection among women who seroconverted to HIV postnatally

\begin{tabular}{|c|c|c|c|c|c|c|}
\hline & $\begin{array}{l}\text { Last negative } \\
\text { ELISA }\end{array}$ & $0-30$ days & $31-90$ days & $91-180$ days & $181-270$ days & 271-365 days \\
\hline \multicolumn{7}{|l|}{ Plasma } \\
\hline $\mathrm{N}$ & 207 & 18 & 125 & 150 & 117 & 63 \\
\hline Proportion of samples with detectable levels of HIV RNA (\%) & 19.32 & 88.89 & 92.00 & 86.67 & 92.31 & 95.24 \\
\hline $\begin{array}{l}\text { Median (IQR) HIV load among samples with detectable levels } \\
\left.\text { of virus ( } \log _{10} \text { copies } / \mathrm{mL}\right)\end{array}$ & $4.97(3.50-5.84)$ & $4.65(4.19-5.41)$ & $4.70(4.06-5.33)$ & $4.44(3.68-5.07)$ & $4.24(3.59-4.81)$ & $4.06(3.69-4.70)$ \\
\hline \multicolumn{7}{|l|}{ Breast milk supernatant } \\
\hline $\mathrm{N}$ & 61 & 8 & 56 & 43 & 23 & 12 \\
\hline Proportion of samples with detectable levels of HIV RNA (\%) & 14.75 & 50.00 & 37.50 & 37.21 & 34.78 & 25.00 \\
\hline $\begin{array}{l}\text { Median (IQR) HIV load among samples with detectable levels } \\
\left.\text { of virus ( } \log _{10} \text { copies } / \mathrm{mL}\right)\end{array}$ & $2.16(1.86-3.10)$ & $4.34(3.61-4.77)$ & $2.03(1.63-2.87)$ & $1.54(1.15-1.80)$ & $1.37(1.34-1.80)$ & $1.45(1.42-2.11)$ \\
\hline
\end{tabular}

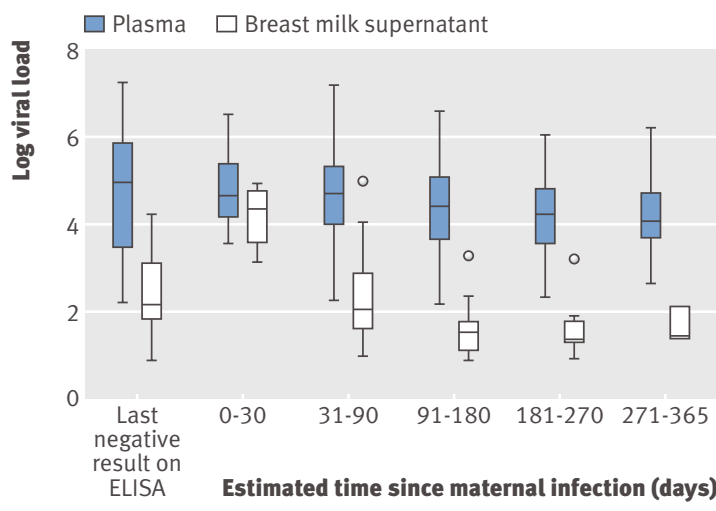

Fig $3 \mid$ Plasma (blue) and breast milk supernatant (open) HIV RNA concentration according to estimated time since HIV infection among women who seroconverted to HIV positive postnatally. Box plots show median, interquartile range, $95 \%$ confidence limits, and outliers. Only samples with detectable HIV RNA load are represented

reduce mother to child transmission among women identified as HIV positive antenatally but will probably reach few seroconverting women.

Breastfeeding associated transmission was 3.8 times higher among infants who were less than 9 months of age at the time of their mother's primary infection than in infants who were older when their mothers acquired HIV. This higher risk for younger infants is in contrast

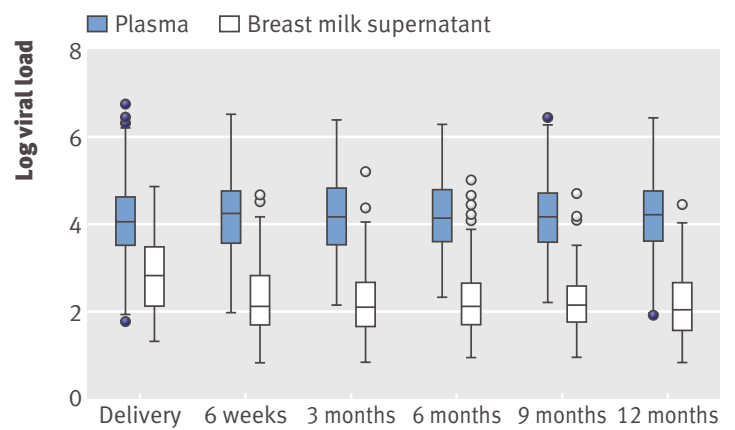

Estimated time since delivery

Fig 4 | Plasma (blue) and breast milk supernatant (open) HIV RNA concentration according to estimated time since delivery among women who tested HIV positive at baseline. Box plots show median, interquartile range, $95 \%$ confidence limits, and outliers. Only samples with detectable HIV RNA load are represented

to the constant risk of breastfeeding associated transmission across infant age reported in chronically infected mothers ${ }^{21}$ and suggests synergism between exposure to very high breast milk HIV load and immature intestinal immune function in facilitating breastfeeding associated HIV transmission. ${ }^{22}$

In this study, cumulative breastfeeding associated transmission was $25 \%$ to $30 \%$ among women who

Table 5 | Proportion of specimens with detectable levels of HIV RNA, and median (IQR) HIV load, according to estimated time since HIV infection among women who among women who tested HIV positive at baseline

\begin{tabular}{|c|c|c|c|c|c|c|}
\hline & Delivery & 6 weeks & 3 months & 6 months & 9 months & 12 months \\
\hline \multicolumn{7}{|l|}{ Plasma } \\
\hline $\mathrm{N}$ & 4035 & 486 & 468 & 441 & 425 & 2597 \\
\hline Proportion of samples with detectable level of HIV RNA (\%) & 92.54 & 96.7 & 96.15 & 94.56 & 95.53 & 94.42 \\
\hline $\begin{array}{l}\text { Median (IQR) HIV load among samples with detectable levels } \\
\text { of virus (log10 copies } / \mathrm{mL} \text { ) }\end{array}$ & $4.05(3.52-4.62)$ & $4.24(3.57-4.75)$ & $4.15(3.55-4.81)$ & $4.13(3.60-4.78)$ & $4.15(3.61-4.71)$ & $4.21(3.64-4.77)$ \\
\hline \multicolumn{7}{|l|}{ Breast milk supernatant } \\
\hline $\mathrm{N}$ & 39 & 773 & 738 & 607 & 176 & 117 \\
\hline Proportion of samples with detectable level of HIV RNA (\%) & 82.05 & 46.44 & 34.82 & 32.62 & 30.68 & 47.86 \\
\hline $\begin{array}{l}\text { Median (IQR) HIV load among samples with detectable levels } \\
\text { of virus (log10 copies } / \mathrm{mL} \text { ) }\end{array}$ & $2.79(2.13-3.64)$ & $2.12(1.73-2.83)$ & $2.09(1.68-2.66)$ & $2.13(1.72-2.65)$ & $2.14(1.77-2.58)$ & $2.05(1.59-2.66)$ \\
\hline
\end{tabular}

$\mathrm{N}$ values are total number of samples available for analysis. 
Table $6 \mid$ Proportion of children infected at specific time points following birth

\begin{tabular}{|c|c|c|c|c|c|c|}
\hline & 30 days & 90 days & 180 days & 270 days & 360 days & 720 days \\
\hline Baseline positive $(n=4495)$ & 18.66 (17.47 to 19.92$)$ & 23.67 (22.35 to 25.06$)$ & 26.51 (25.13 to 27.96$)$ & 28.79 (27.34 to 30.28$)$ & 30.51 (29.03 to 32.05$)$ & 35.35 (33.40 to 37.37$)$ \\
\hline Baseline seroconverter $(n=17)$ & 9.09 (1.33 to 49.19$)$ & 27.27 (9.72 to 62.92$)$ & 45.45 (22.04 to 77.15$)$ & 65.91 (38.37 to 90.86$)$ & $65.91(38.37$ to 90.86$)$ & 65.91 (38.37 to 90.86$)$ \\
\hline
\end{tabular}

- HIV transmission among women positive at baseline

- - - HIV transmission or death among women positive at baseline

...-- HIV transmission among baseline seroconverters

- HIV transmission or death among baseline seroconverters
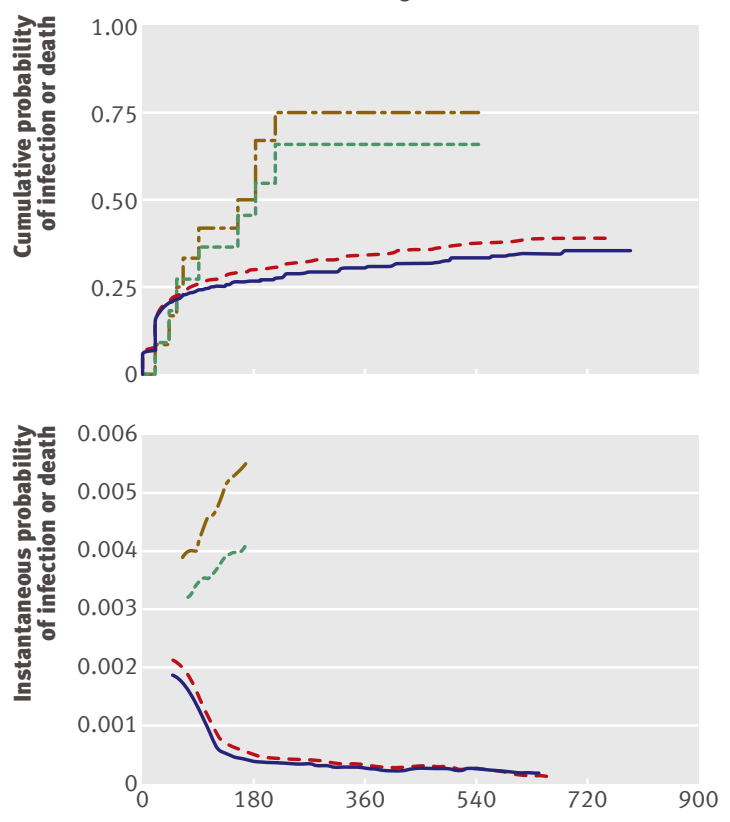

Days of HIV exposure

Fig 5 | Cumulative (top panel) and instantaneous (one day; bottom panel) probability of HIV transmission and HIV transmission or death among baseline positive women and baseline seroconverter women

seroconverted postnatally, about double the proportion among women positive at baseline (14\%). However, unlike the relatively low but continuously accumulating risk of postnatal transmission in baseline positive women ( 9 infections per 100 child years of breast feeding), the risk among postnatal seroconverter women was very high but only for a relatively brief period of time. Of the transmissions among the 51 mothers for whom the timing of infection was known within a 90 day interval, $62 \%$ and $85 \%$ of transmission occurred within three and six months, respectively, of the mothers' infection. Thus, during the first three months after maternal infection, the transmission rate among postnatal seroconverter women was not double but eightfold higher than among the baseline positive women. This substantial variation in the rates of breastfeeding associated transmission among postnatal seroconverter mothers was mirrored by a transient peak in breast milk HIV load in at least some women. During the first 30 days after primary infection in this group, median breast milk HIV load peaked in the same range as plasma HIV load $\left(\sim 4.5 \log _{10}\right)$ in the samples with detectable levels of viral RNA. Over the subsequent two to five months, both the proportion of samples in which viral RNA could be detected (from $50 \%$ to about 30\%) and the HIV loads declined appreciably. During this period, HIV loads were $\sim 1.5 \log _{10}$, about $0.5 \log _{10}$ lower than median concentrations among baseline positive women. In contrast, plasma HIV load declined more slowly in postnatal seroconverter mothers, remaining higher than that of baseline positive women until nine to 12 months after primary infection.

Although the mechanism that underlies this rapid decay of HIV RNA concentrations in breast milk is unknown, it is possible that the characteristics of the cellular fractions of breast milk versus those of blood contribute to this finding. In particular, it is known that the great majority of breast milk $\mathrm{T}^{23-26}$ and $\mathrm{B}^{27}$ cells are activated or antigen primed, compared with only about half of their counterpart cell populations in peripheral blood. The activation state of immune cells in the mammary gland might plausibly influence breast milk HIV loads, either by direct viral control or by "purging" of latently infected cells in the mammary tissues.

Among the baseline positive women in ZVITAMBO, breastfeeding associated transmission was highly correlated with indicators of advanced maternal disease (low CD4 count, low haemoglobin concentration, low midupper arm circumference, and death within the postpartum year). ${ }^{12}$ Taken together, our analyses confirm that the risk of breastfeeding associated transmission follows a U-shaped curve similar to that reported for heterosexual transmission ${ }^{22829}$ : it is high during primary infection, low during the latency period of HIV, and then high again during advanced disease.

\section{Strengths and limitations of study}

The primary strength of this study is the large number of postnatal seroconverter women followed at three monthly intervals together with their breastfeeding infants. The inclusion of a concurrent large cohort of baseline positive women with breastfeeding infants known to have escaped intrauterine and intrapartum infection provides an important comparison group. In addition, plasma and breast milk HIV load data were available from baseline positive women throughout the postnatal period and from postnatal seroconverter women during and following primary HIV infection. Finally, the study included a group of 17 mother-infant pairs in which the mother's blood sample collected soon after delivery tested HIV negative with ELISA and HIV positive with PCR. To our knowledge this is the first report of the transmission risk for women who give birth during HIV primary infection. 


\section{WHAT IS ALREADY KNOWN ON THIS TOPIC}

Primary HIV infection (the interval between infection and seroconversion, when detectable concentrations of HIV specific antibodies have developed) is the period in which a large proportion of all new sexually acquired HIV infections are transmitted

Primary HIV infection is also implicated in high levels of breastfeeding associated mother to child transmission of HIV during the lactation period, although empirical data are limited

\section{WHAT THIS STUDY ADDS}

Breastfeeding associated transmission of HIV is high during maternal primary HIV infection, and is mirrored by a high but transient peak in breast milk HIV load

About $20 \%$ of all breastfeeding associated transmissions may occur among infants whose mothers seroconverted postnatally

Almost two thirds of breastfeeding associated transmission among women who seroconvert postnatally may occur during the first 90 days after maternal infection, when the mother may still test negative for HIV according to antibody based diagnostic tests

A limitation of this study is the relatively small numbers of breast milk samples available from seroconverting mothers during the first month after infection. However, the consistency between breast milk HIV load and risk of instantaneous transmission suggests that the available data on breast milk HIV load are representative. A second limitation of the study is that it was not designed to measure breastfeeding associated transmission between delivery and six weeks post partum, given the technical difficulty in distinguishing between late intrauterine, intrapartum, and early postnatal transmission.

\section{Policy implications}

Repeat testing of HIV negative women during antenatal care has been recommended so that women who seroconvert during pregnancy can be given intrapartum antiretroviral prophylaxis. ${ }^{18}$ Our findings indicate that extending this strategy of repeat testing to the postpartum period would have only a modest effect on breastfeeding associated HIV transmission. Among postnatal seroconverter women with a short seroconversion interval enrolled in this trial, $62 \%$ transmitted HIV to their infant in the first three months after maternal infection. Given that the window period for ELISA and rapid HIV tests can be as long as several weeks, the majority of transmissions that will occur among postnatal seroconverter women will have already happened by the time the mothers test HIV positive. For this reason, eliminating paediatric HIV infection will require renewed focus on prevention of primary HIV, particularly among pregnant and breastfeeding women.

Members of the ZVITAMBO study group, in addition to the named authors, are: John Hargrove, Agnes Mahomva, Florence Majo, Michael Mbizvo, Faith Mzengeza, Mary Ndhlovu, Lidia Propper, Phillipa Rambanepasi, Andrea Ruff, and Clare Zunguza.

Contributors: JHH was the principal investigator of the ZVITAMBO trial, drafted this manuscript, had access to the data, and controlled the decision to publish. $\mathrm{JHH}$ also acts as guarantor for the work. EM and LHM designed and conducted the statistical analyses. KM managed all laboratory work. RN and HC designed and implemented the data management system, including calculation of laboratory data. PJI, KJN, NT, EGP, and BJW contributed to the design, implementation, and interpretation of the ZVITAMBO trial. All authors contributed to interpreting the data presented in this paper and to writing this manuscript.
Funding: The ZVITAMBO Project was supported by the Canadian International Development Agency (R/C Project 690/M3688); United States Agency for International Development (USAID; cooperative agreement number HRN-A-00-97-00015-00 between Johns Hopkins University and the Office of Health and Nutrition-USAID); a grant from the Bill and Melinda Gates Foundation, Seattle, WA; the SARA Project, operated by the Academy for Educational Development, Washington, DC and the Department for International Development, United Kingdom ("Saving Maternal and Newborn Lives in the Context of HIV and AIDS in Zimbabwe" Grant No AG 4996 (MIS code 073-555-013 CA 007)). The sponsors of the study had no role in the study design, recruitment of participants, data collection, data analysis, data interpretation, writing of the report, or the decision to submit for publication. The corresponding author had full access to all data and had final responsibility for the decision to submit for publication.

Competing interests: All authors have completed the Unified Competing Interest form at www.icmje.org/coi_disclosure.pdf (available on reques from the corresponding author) and declare: no support from any organisation for the submitted work; no financial relationships with any organisations that might have an interest in the submitted work in the previous 3 years; no other relationships or activities that could appear to have influenced the submitted work.

Ethical approval: The Medical Research Council of Zimbabwe, the Medicines Control Authority of Zimbabwe, Johns Hopkins Bloomberg School of Public Health Committee on Human Research, and the Research Institute of the McGill University Health Centres Research Ethics Committee approved the study protocol.

Data sharing: Data are available for secondary analyses under some circumstances; please send requests to corresponding author at jhumphrey@zvitambo.co.zW.

This research was presented in part at the 16th International Al DS Conference, Toronto, Canada; 2006 August 13-18 (poster No MOPE0384)

1 Daar ES, Moudgil T, Meyer RD, Ho DD. Transient high levels of viremia in patients with primary human immunodeficiency virus type 1 infection. N Engl J Med 1991;324:961-4.

2 Wawer MJ, Gray RH, Sewankambo NK, Serwadda D, Li X, Laeyendecker O, et al. Rates of HIV-1 transmission per coital act, by stage of HIV-1 infection, in Rakai, Uganda. J Infect Dis 2005;191:1403-9.

3 Brenner BG, Roger M, Routy JP, Moisi D, Ntemgwa M, Matte C, et al. High rates of forward transmission events after acute/early HIV-1 infection. J Infect Dis 2007;195:951-9.

4 Colebunders R, Kapita B, Nekwei W, Bahwe Y, Lebughe I, Oxtoby M, et al. Breastfeeding and transmission of HIV. Lancet 1988;2:1487.

5 Hira SK, Mangrola UG, Mwale C, Chintu C, Tembo G, Brady WE, et al. Apparent vertical transmission of human immunodeficiency virus type 1 by breast-feeding in Zambia. J Pediatr 1990;117:421-4.

6 Palasanthiran P, Ziegler JB, Stewart GJ, Stuckey M, Armstrong JA, Cooper DA, et al. Breast-feeding during primary maternal human immunodeficiency virus infection and risk of transmission from mother to infant. J Infect Dis 1993;167:441-4.

7 Van de Perre P, Simonon A, Msellati P, Hitimana DG, Vaira D, Bazubagira A, et al. Postnatal transmission of human immunodeficiency virus type 1 from mother to infant. A prospective cohort study in Kigali, Rwanda. N Engl J Med 1991;325:593-8.

8 Van de Perre P, Hitimana DG, Simonon A, Dabis F, Msellati P, Karita P, et al. Postnatal transmission of HIV-1 associated with breast abscess. Lancet 1992;339:1490-1.

9 Dunn DT, Newell M-L, Ades AE, Peckham CS. Risk of human immunodeficiency virus type 1 transmission through breastfeeding. Lancet 1992;340:585-8.

10 Liang K, Gui X-E, Zhang YZ, Zhuang K, Meyers K, Ho DD. A case series of 104 women infected by HIV-1 via blood transfusion postnatally: high rate of HIV-1 transmission to infants through breastfeeding. J Infect Dis 2009;200:682-6.

11 Humphrey JH, Iliff PJ, Marinda E, Mutasa K, Moulton LH, Chidawanyika $\mathrm{H}$, et al. Effects of a single large dose of vitamin $\mathrm{A}$, given during the postpartum period to HIV-positive women and their infants, on child HIV infection, HIV-free survival, and mortality.J Infect Dis 2006;193:860-71.

12 Iliff PJ, Piwoz EG, Tavengwa NV, Zunguza CD, Marinda ET, Nathoo KJ, et al. Early exclusive breastfeeding reduces the risk of postnatal HIV1 transmission and increases HIV-free survival. AIDS 2005;19:699-708.

13 Humphrey JH, Hargrove JW, Malaba LC, Iliff PJ, Moulton LH, Mutasa K, et al. HIV incidence among post-partum women in Zimbabwe: risk factors and the effect of vitamin A supplementation. AIDS 2006;20:1437-46 
14 World Health Organization. HIV and infant feeding: new evidence and programmatic experience. Report of a technical consultation. WHO, 2006.

15 Humphrey JH, Nathoo KJ, Hargrove JW, Iliff PJ, Mutasa KE, Moulton LH, et al. HIV-1 and HIV-2 prevalence and associated risk factors among postnatal women in Harare, Zimbabwe. Epidemiol Infect 2007;135:933-42

16 Tavengwa NV, Piwoz EG, Iliff PJ, Moulton LH, Zunguza CD, Nathoo KJ, et al. Adoption of safer infant feeding and postpartum sexual practices and their relationship to maternal HIV status and risk of acquiring HIV in Zimbabwe. Trop Med Int Health 2007;12:97-106.

17 Piwoz EG, Iliff PJ, Tavengwa NV, Gavin L, Marinda E, Lunney K, et al. An education and counselling program for preventing breastfeedingassociated HIV transmission in Zimbabwe: design and impact on maternal knowledge and behaviour. J Nutr 2005;135:950-5.

18 Moodly D, Esterhuizen TM, Pather T, Chetty V, Ngaleka L. High HIV incidence during pregnancy: compelling reason for repeat HIV testing. AIDS 2009;23:1255-9.

19 Kieffer MP, Nhlabatsi B, Mahdi M. Addressing missed opportunities for PMTCT in maternity reduces perinatal HIV transmission in Swaziland: preliminary study results show increases in ARV access and uptake [abstract TUPEC051]. 5th Conference on $\mathrm{H}$ IV Pathogenesis, Treatment and Prevention, Cape Town, South Africa; 2009 July 19-23.

20 World Health Organization. Rapid advice: antiretroviral therapy fo HIV infection in adults. WHO, 2009.

21 Coutsoudis A, Dabis F, Fawzi W, Gaillard P, Haverkamp G, Harris DR, et al. Late postnatal transmission of HIV-1 in breast-fed children: an individual patient data meta-analysis. I Infect Dis 2004;189:2154-66
22 Insoft RM, Sanderson IR, Walker WA. Development of immune function in the intestine and its role in neonatal disease. Pediatr Clin North Am 1996;43:551-71.

23 Bertotto A, Castellucci G, Scalise F, Tognellini R, Vaccaro R. "Memory" T cells in human breast milk. Acta Paediatr Scand 1991;80:98-9.

24 Bertotto A, Gerli R, Fabietti G, Crupi S, Arcangeli C, Scalise F, et al. Human breast milk T lymphocytes display the phenotype and functional characteristics of memory T cells. Eur J Immunol 1990;20:1877-90.

25 Bertotto A, Castellucci G, Scalise F, Vaccaro R. Human milk T lymphocytes are mostly HML-1-positive cells. Eur J Pediatr 1992;151:150.

26 Wirt DP, Adkins LT, Palkowetz KH, Schmalsteig FC, Goldman AS. Activated and memory T lymphocytes in human milk. Cytometry 1992;13:282-90.

27 Tuaillon E, Valea D, Becquart P, Al Tabaa Y, Meda N, Bollore K, et al. Human milk-derived B cells: a highly activated switched memory cell population primed to secrete antibodies. J Immunol 2009;182;7155-62.

28 De Vincenzi I. European study group on heterosexual transmission of HIV. A longitudinal study of human immunodeficiency virus transmission by heterosexual partners. $N$ Engl J Med 1994;331:341-6.

29 Leynaert B, Down AM, de Vincenzi I. European study group on heterosexual transmission of HIV, heterosexual transmission of human immunodeficiency virus: variability of infectivity throughout the course of infection. Am J Epidemiol 1998;148:88-96.

Accepted: 22 September 2010 